\title{
Nonlinear Static and Dynamic Stiffness Characteristics of Support Hydraulic System of TBM
}

\author{
Jianfeng Tao ${ }^{1}$, Junbo Lei ${ }^{1 *}$, Chengliang Liu ${ }^{1}$ and Wei Yuan ${ }^{2}$
}

\begin{abstract}
Full-face hard rock tunnel boring machines (TBM) are essential equipment in highway and railway tunnel engineering construction. During the tunneling process, TBM have serious vibrations, which can damage some of its key components. The support system, an important part of TBM, is one path through which vibrational energy from the cutter head is transmitted. To reduce the vibration of support systems of TBM during the excavation process, based on the structural features of the support hydraulic system, a nonlinear dynamical model of support hydraulic systems of TBM is established. The influences of the component structure parameters and operating conditions parameters on the stiffness characteristics of the support hydraulic system are analyzed. The analysis results indicate that the static stiffness of the support hydraulic system consists of an increase stage, stable stage and decrease stage. The static stiffness value increases with an increase in the clearances. The pre-compression length of the spring in the relief valve affects the range of the stable stage of the static stiffness, and it does not affect the static stiffness value. The dynamic stiffness of the support hydraulic system consists of a U-shape and reverse U-shape. The bottom value of the U-shape increases with the amplitude and frequency of the external force acting on the cylinder body, however, the top value of the reverse U-shape remains constant. This study instructs how to design the support hydraulic system of TBM.
\end{abstract}

Keywords: Tunnel boring machine, Support hydraulic system, Nonlinear model, Static stiffness characteristics, Dynamic stiffness characteristics

\section{Introduction}

Full-face hard rock tunnel boring machines (TBM) are widely used in highway and railway tunnel engineering construction because excavating tunnels with TBM has advantages such as little environmental disturbance, high tunnel efficiency, low personnel expenditure, and better working conditions and safety $[1,2]$. The basic elements of TBM have serious vibrations because the load changes suddenly due to the rock being broken in a working environment of high hardness, quartz content, and surface stress. The system vibrations generate noise, harm workers' health, and may damage key components of the system [3-6]. The vibration energy is transferred to the

\footnotetext{
*Correspondence: jblei@sjtu.edu.cn

${ }^{1}$ School of mechanical engineering, Shanghai Jiaotong University, Shanghai 200240, China

Full list of author information is available at the end of the article
}

support system through the main beam, making the support system vibrate [7]. As a key working component of TBM, the support system provides the excavating counter-torque and counter-force during the excavation process $[8,9]$, which has an important influence on system vibration; therefore, the stiffness characteristics of the support system receive significant concern $[10,11]$.

Support system stiffness is composed of hydraulic system stiffness, mechanical structure stiffness, and contact stiffness $[12,13]$. Wu et al. [14] established a multi-coupling dynamic model for support systems of TBM and carried out a multi-position dynamic strain test based on practical engineering to calibrate the dynamic model. They pointed out that the support directly affected the tunneling efficiency of TBM. Yu et al. [15] studied the relationship between the roughness and fractal parameters of three dimensional tunnel surfaces based on fractal theory and found that 
increases in the fractal dimension, elastic modulus, normal loads, and number of support boots cause an increase in the interfacial contact stiffness, and an increase in the ratio of soft rocks in the contacting area causes an increase in the contact stiffness. This shows that the contact stiffness is influenced by the support hydraulic system because the normal loads of support shoes can be adjusted by the support hydraulic system. Hence, hydraulic system stiffness characteristics play an important role in the support system [16, 17]. Wu et al. [18] obtained the static stiffness of gripper cylinders and the machine-hydraulic-rock stiffness through the linearization method near the equilibrium point of the simplified model. The dynamic characteristics of the relief valve in the support hydraulic system and the leakage characteristics of the gripper cylinder had not been taken into account in the system model, and linearization may lack some important characteristics. Wang et al. [19] studied the nonlinear dynamic characteristics of moving cylinders by theoretical analysis and experimental verification, and they found that the cylinder took on soft spring and hard spring properties in different working situations; however, this research merely focused on the nonlinear stiffness characteristics of the hydraulic cylinder, and the stiffness characteristics of the hydraulic system and the leakage characteristics of the cylinder were not investigated. Lei et al. [20] analyzed the gripper cylinder internal leakage characteristics of TBM and the influences of the cylinder structure parameters and working conditions parameters on the structure feature of the support hydraulic system. The relief valve model in this research was simplified as a linear relation between input pressure and flowrate, and the dynamic characteristics of the relief valve were neglected. As for the support hydraulic system, the stiffness characteristics depend heavily on the open and close of the relief valve and the dynamic characteristics of the valve. In fact, the static and dynamic characteristics of the poppet relief valve have a significant influence on the efficiency of the hydraulic system [21, 22]. For this purpose, it is necessary to investigate the nonlinear static and dynamic stiffness characteristics of the support hydraulic system while also considering the dynamic characteristics of the relief valve and working conditions.

In this paper, taking into account the dynamic model of the relief valve, the nonlinear dynamic model of the support hydraulic system is established according to the structural feature of the support hydraulic system, and the influences of the component structure parameters and operating conditions parameters on the stiffness characteristics of the support hydraulic system are analyzed.

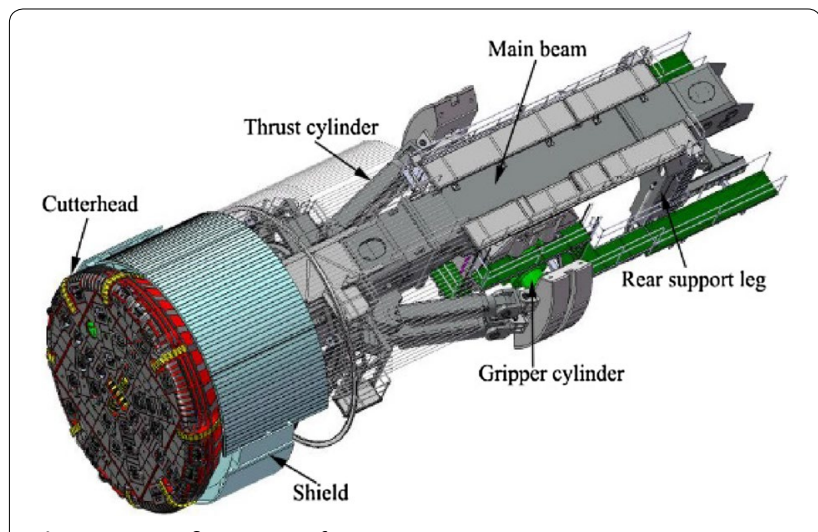

Figure 1 Configuration of TBM

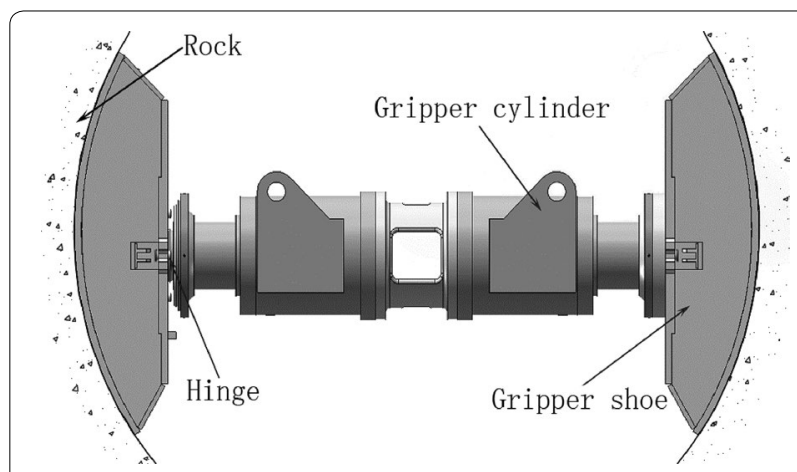

Figure 2 Schematic of support system

\section{Configuration of the Support Hydraulic System}

TBM are composed of a main beam, cutterhead, gripper cylinder, shield, rear support leg, and thrust cylinder as shown in Figure 1 [23, 24].

During the excavation process, gripper shoes support the rock, relying on the output force of the gripper cylinder to provide the counter-force and counter-torque needed for excavating, and Figure 2 shows the working state of an gripper cylinder [25].

Based on Figure 2, the support system in TBM includes gripper shoes, hinges, and a support hydraulic system, and the support hydraulic system in the working state is demonstrated in Figure 3.

As shown in Figure 3, the support hydraulic system consists of two gripper cylinders, two check valves, and two relief valves. The two gripper cylinders are fixed together; their rod-less chambers are connected, and the two relief valves are connected to the rod chambers, The rod-less chambers of the gripper cylinders are connected with a pipeline. The support hydraulic system can be simplified and illustrated in Figure 4. 

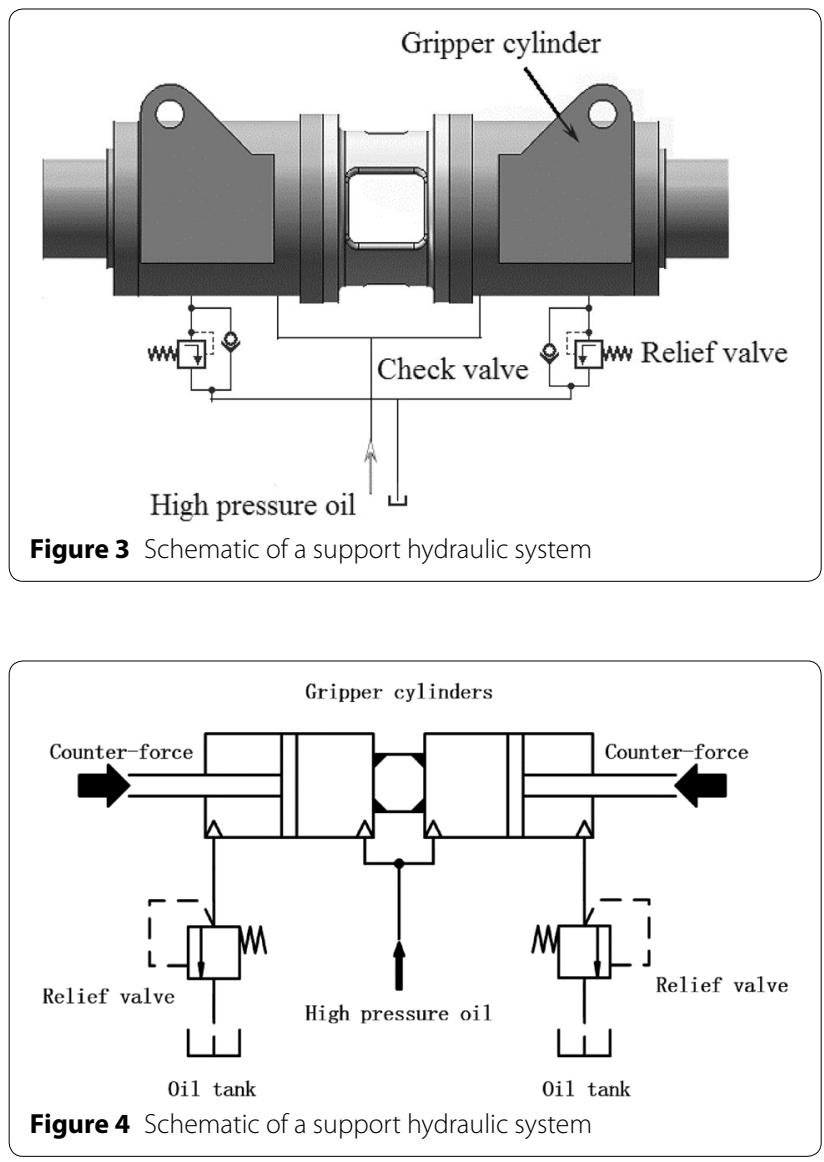

\section{Dynamic Model of Support Hydraulic System}

As shown in Figure 4, the dynamic model of the support hydraulic system consists of the gripper cylinder sub model and the relief valve sub model. The gripper cylinder sub model includes the moment equation and the leakage equation of the cylinder, the relief valve sub model includes the flow model and the force equilibrium equation of the valve core.

\subsection{Dynamic Model of Gripper Cylinder}

As shown in Figure 5 and taking the cylinder body as the research object and neglecting the viscous force, the following equation can be obtained based on Newton second theorem.

$$
m_{c} \ddot{x}=\left(p_{2}-p_{1}\right) A_{c}+F_{l c}-F_{f c} \operatorname{sign}(\dot{x}),
$$

where $m_{c}$ is the mass of the cylinder body, $x$ is the displacement of the cylinder body, $A_{c}$ denotes the effective area of the rod chamber, $F_{l c}$ stands for the external force acting on the cylinder body, $F_{f c}$ is the friction between the cylinder body and the piston, $p_{1}$ and $p_{2}$ are the rod chamber of the two cylinders, respectively, and $\ddot{x}, \dot{x}$

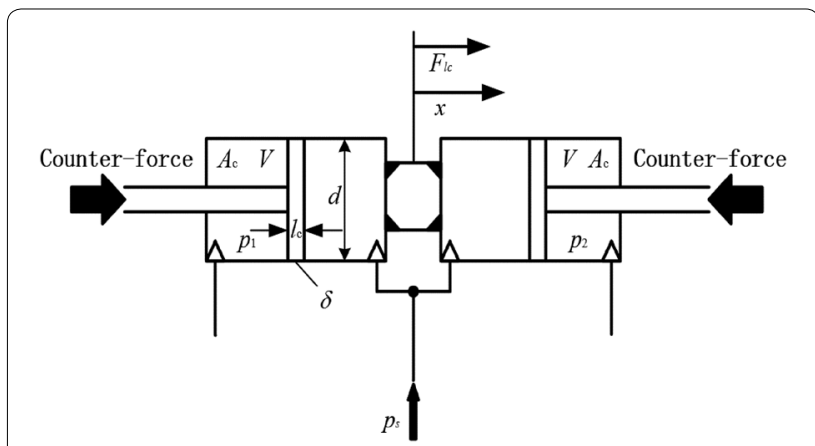

Figure 5 Schematic of a support hydraulic system

are the acceleration and velocity of the cylinder body, respectively.

According to the pressure-rise rate equation, the pressure expression of the rod chamber can be obtained [26].

$$
\begin{aligned}
& \dot{p}_{1}=\frac{\beta_{e}}{V-x A_{c}}\left(q_{l 1}+\dot{x} A_{c}-q_{v 1}\right), \\
& \dot{p}_{2}=\frac{\beta_{e}}{V+x A_{c}}\left(q_{l 2}-\dot{x} A_{c}-q_{v 2}\right),
\end{aligned}
$$

where $\beta_{e}$ is the bulk modulus of the working fluid media, $V$ is the initial volume of the rod chamber, $q_{l 1}$ and $q_{l 2}$ are the leakage flowrates from the rod-less chambers to the rod chambers of the two cylinders, respectively, and $q_{v 1}$, $q_{v 2}$ denote the flowrates of the relief valves.

Based on the Navier-Stokes equations and neglecting the eccentricity of the piston in the gripper cylinder, the volume rate of flow between the rod-less chamber and the rod chamber is derived as [27]

$$
\begin{aligned}
& q_{l 1}=\frac{\pi d \delta_{1}^{3}\left(p_{s}-p_{1}\right)}{12 \mu l_{c}}-\frac{\pi d \delta_{1}}{2} \dot{x}, \\
& q_{l 2}=\frac{\pi d \delta_{2}^{3}\left(p_{s}-p_{2}\right)}{12 \mu l_{c}}+\frac{\pi d \delta_{2}}{2} \dot{x},
\end{aligned}
$$

where $d$ denotes the diameter of the cylinder piston, $\delta_{1}$ and $\delta_{2}$ are the clearances between the piston and the internal wall of the two cylinders, respectively, $p_{s}$ is the pressure in the rod-less chamber, $\mu$ is the absolute viscosity of the working fluid media, and $l_{c}$ stands for the length of the piston.

\subsection{Dynamic Model of Relief Valve}

The dynamic characteristics of the relief valve play an important role in the stiffness characteristics of the support hydraulic system. Figure 6 shows the schematic of the relief valve in the open position [28]. 


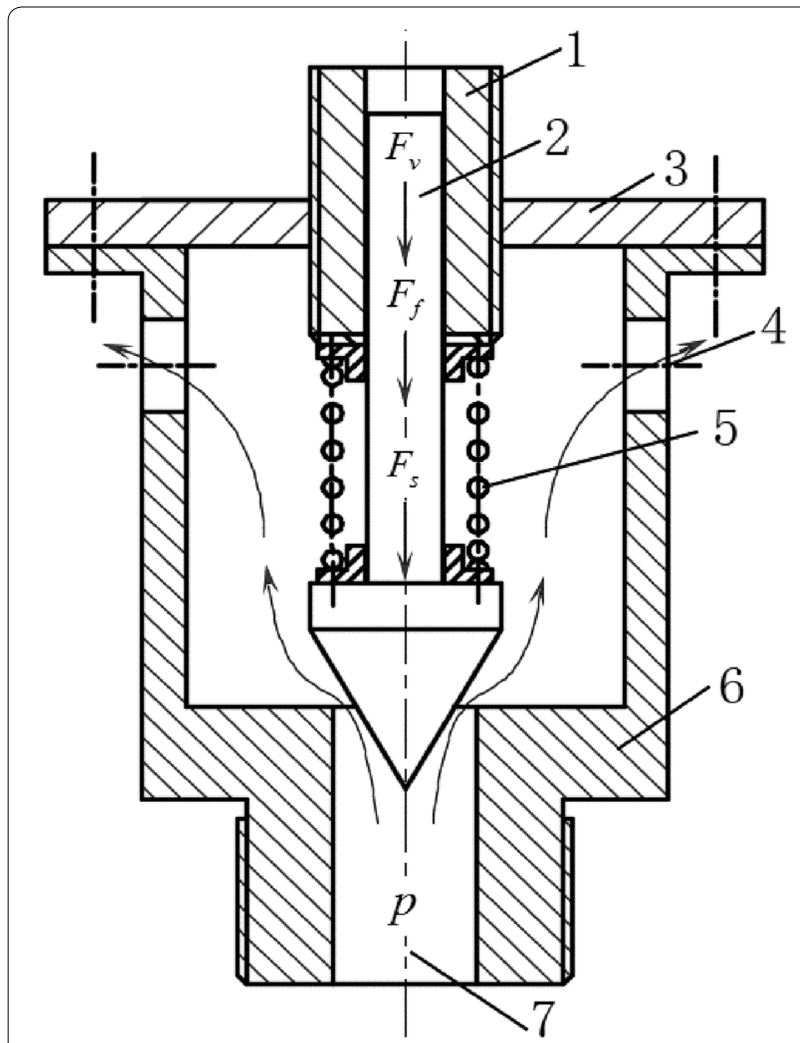

Figure 6 Schematic of a support hydraulic system. 1. Pre-compression thread, 2. Valve core, 3. Valve cover, 4. Outlet, 5. Spring, 6. Valve body, 7. Inlet

As shown in Figure 6, the relief valve consists of a precompression thread, valve cover, valve core, spring, and valve body.

\subsubsection{Flow Equation for Relief Valve}

The flow through the orifice can be expressed as [29]

$$
q=C_{d} A(y) \sqrt{\frac{2 p}{\rho}}
$$

where $C_{d}$ is the discharge coefficient, $A(y)$ is the flowthrough area, $y$ is the position of the valve body, $p$ is the pressure difference between the inlet and the outlet of the relief valve, and $\rho$ is the density of the fluid media.

Figure 7 shows the geometry of the flow area, and the flow-through area can be approximately given as [26]

$$
A(y)=\pi D_{s} \sin \theta y,
$$

where $D_{s}$ is the diameter of the seat port of the relief valve, and $\theta$ denotes the half angle of the valve core.

Based on Bazsó's [30] experimental results, Eq. (6) can be rewritten as

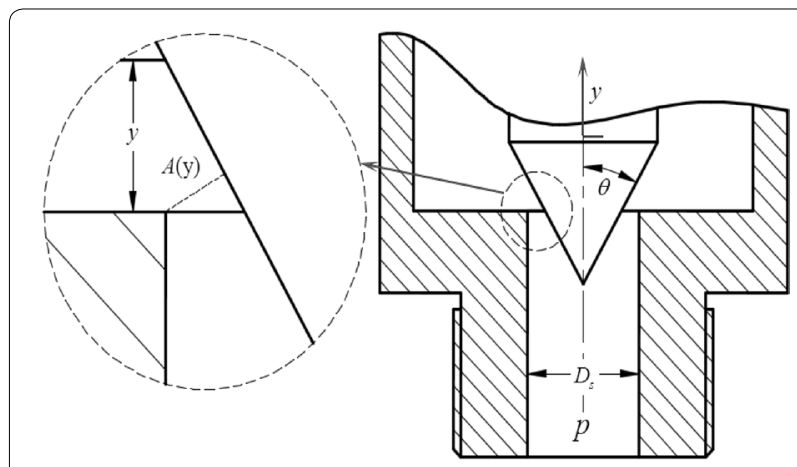

Figure 7 Geometry of the flow area

$$
q=0.1239 \pi D_{s} v\left(\frac{y \sin \theta}{v} \sqrt{\frac{2 p}{\rho}}\right)^{1.3086}
$$

where $v$ denotes the kinematic viscosity of the fluid media.

\subsubsection{Mechanical Analysis of Relief Valve Core}

The forces acting on the valve core, as shown in Figure 6, include the friction force, spring force, viscous force, and flow force, which are represented symbolically as $F_{f}, F_{s}$, $F_{v}$ and $F_{f l}$, respectively. The spring force and friction force are given by

$$
\begin{aligned}
& F_{s}=-k\left(y_{0}+y\right), \\
& F_{v}=-B_{v} \dot{y},
\end{aligned}
$$

where $k$ is the stiffness of the spring, $y_{0}$ is the initial precompression length of the spring, $B_{v}$ is the damping coefficient, and $\dot{y}$ denotes the velocity of the valve body.

The flow forces depend on the flowrate through the orifice and the inlet pressure, and they are an important part of the forces acting on the valve core. Figure 8 shows the

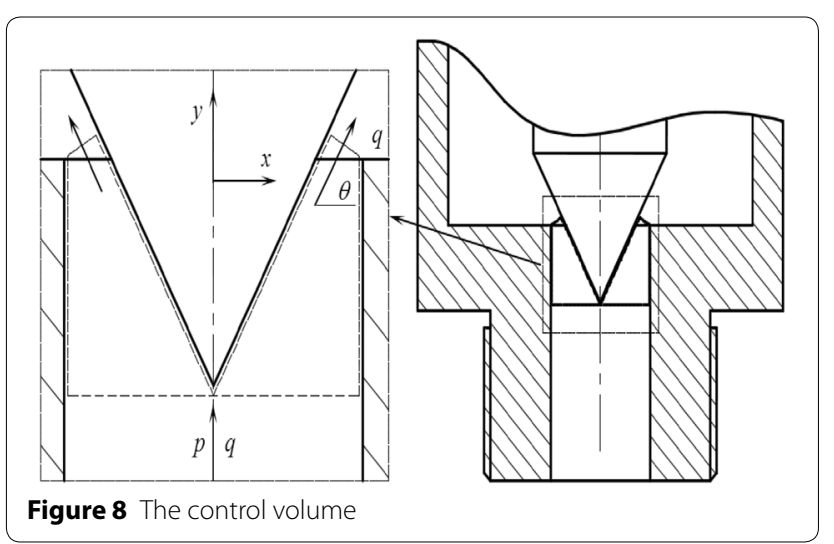


control volume for calculating the flow forces acting on the valve core.

According to the Reynolds transport theorem, the $Y$-component of the fluid force acting on the valve core can be expressed as [27, 28, 31]

$$
\begin{aligned}
F_{y}= & p A_{s}-\rho\left[\left(q-\dot{y} A_{s}\right)^{2}\left(\frac{\cos \theta}{A(y)}-\frac{1}{A_{s}}\right)\right. \\
& \left.+\left(\dot{q}-\ddot{y} A_{s}\right)\left(\frac{D_{s}}{2 \tan \theta}-y\right)-\left(q-\dot{y} A_{s}\right) \dot{y}\right], \quad y>0, \\
F_{y}= & p A_{s}-\rho\left(\dot{q}-\ddot{y} A_{s}\right) \frac{D_{s}}{2 \tan \theta}, \quad y=0,
\end{aligned}
$$

where $F_{y}$ stands for the component force in the $y$ direction, $\ddot{y}$ denotes the acceleration of the valve core, and $A_{s}$ is the seat area.

Based on Newton's second theorem, the following equation can be obtained:

$$
m_{v} \ddot{y}=F_{y}-k\left(y_{0}+y\right)-F_{f} \operatorname{sign}(\dot{y})-F_{v},
$$

where $m_{\nu}$ is the mass of the valve core.

Substituting Eqs. (10) and (11) into Eq. (12), under the condition that $y>0$, the kinematic equation of the valve body takes the form

$$
\begin{aligned}
m_{v} \ddot{y}= & p A_{s}+\rho\left[\left(q-\dot{y} A_{s}\right)^{2}\left(\frac{1}{A_{s}}-\frac{\cos \theta}{A(y)}\right)\right. \\
& \left.-\left(\dot{q}-\ddot{y} A_{s}\right)\left(\frac{D_{s}}{2 \tan \theta}-y\right)+\left(q-\dot{y} A_{s}\right) \dot{y}\right] \\
& -k\left(y_{0}+y\right)-F_{f} \operatorname{sign}(\dot{y})-B_{v} \dot{y} .
\end{aligned}
$$

Therefore, the dynamic model of the relief valve can be expressed by Eqs. (8) and (13).

\subsection{Dynamic Model of Support Hydraulic System}

Based on the sub-models of the gripper cylinder and relief valve and neglecting the pipe length between the cylinder and the relief valve, the volume flowrate through the relief valves can be expressed as follows:

$$
\begin{aligned}
& q_{v 1}=0.1239 \pi D_{s} v\left(\frac{y_{1} \sin \theta}{v} \sqrt{\frac{2 p_{1}}{\rho}}\right)^{1.3086}, \\
& q_{v 2}=0.1239 \pi D_{s} v\left(\frac{y_{2} \sin \theta}{v} \sqrt{\frac{2 p_{2}}{\rho}}\right)^{1.3086},
\end{aligned}
$$

where $y_{1}$ and $y_{2}$ are the displacements of the relief valve cores.

The dynamic model of the relief valve can be given as follows:

$$
\begin{aligned}
m_{v} \ddot{y}_{1}= & p_{1} A_{s}+\rho\left[\left(q_{v 1}-\dot{y}_{1} A_{s}\right)^{2}\left(\frac{1}{A_{s}}-\frac{\cos \theta}{A\left(y_{1}\right)}\right)\right. \\
& \left.+\left(q_{v 1}-\dot{y}_{1} A_{s}\right) \dot{y}_{1}-\left(\dot{q}_{v 1}-\ddot{y}_{1} A_{s}\right)\left(\frac{D_{s}}{2 \tan \theta}-y_{1}\right)\right] \quad y>0, \\
& -k\left(y_{0}+y_{1}\right)-F_{f} \operatorname{sign}\left(\dot{y}_{1}\right)-B_{v} \dot{y}_{1}, \\
m_{v} \ddot{y}_{1}= & p_{1} A_{s}-\rho\left(\dot{q}_{v 1}-\ddot{y}_{1} A_{s}\right) \frac{D_{s}}{2 \tan \theta}-k\left(y_{0}+y_{1}\right) \quad y=0, \\
& -F_{f} \operatorname{sign}\left(\dot{y}_{1}\right)-B_{v} \dot{y}_{1}, \\
m_{v} \ddot{y}_{2}= & p_{2} A_{s}+\rho\left[\left(q_{v 2}-\dot{y}_{1} A_{s}\right)^{2}\left(\frac{1}{A_{s}}-\frac{\cos \theta}{A\left(y_{2}\right)}\right)\right. \\
& \left.-\left(\dot{q}_{v 2}-\ddot{y}_{2} A_{s}\right)\left(\frac{D_{s}}{2 \tan \theta}-y_{2}\right)+\left(q_{v 2}-\dot{y}_{2} A_{s}\right) \dot{y}_{2}\right] \quad y>0, \\
& -k\left(y_{0}+y_{2}\right)-F_{f} \operatorname{sign}\left(\dot{y}_{2}\right)-B_{v} \dot{y}_{2}, \\
m_{v} \ddot{y}_{2}= & p_{2} A_{s}-\rho\left(\dot{q}_{v 2}-\ddot{y}_{2} A_{s}\right) \frac{D_{s}}{2 \tan \theta}-k\left(y_{0}+y_{2}\right) \quad y=0 . \\
& -F_{f} \operatorname{sign}\left(\dot{y}_{2}\right)-B_{v} \dot{y}_{2},
\end{aligned}
$$

Therefore, the dynamic model of support hydraulic system can be described by Eqs. (1)-(5) and (13)-(17).

\section{Nonlinear Stiffness Characteristics Analysis}

Based on the dynamic model of the support hydraulic system, the simulation model was established the Simulink package in MATLAB. The parameters employed in the simulation model are listed in Table 1.

Defining the support hydraulic system stiffness according to the simulation model as $k_{s}=F_{l c} / x$, the nonlinear static and dynamic stiffness characteristics of the support hydraulic system are analyzed as follows. In static stiffness analysis, a single direction of $F_{l c}$ is investigated for simplicity because the support hydraulic system is symmetrical.

\subsection{Quasi-static Stiffness Characteristics Analysis 4.1.1 Influence of the Clearances on Static Stiffness}

The velocity of the gripper cylinder body is neglected when calculating static stiffness. The influences of the clearances between the piston and the internal wall of the cylinder on static stiffness is analyzed.

For $\delta_{1}=\delta_{2}$, the static stiffness characteristic of the support hydraulic system at different conditions is plotted in Figure 9.

Based on Figure 9, under the condition that $\delta_{1}=\delta_{2}$, the static stiffness of the support hydraulic system can be divided into three parts: an increase stage, stable stage, and decrease stage. Static stiffness increases as the external force acting on the cylinder body increases in the increase stage, remains approximately constant in the stable stage, and decreases in the last stage. 
Table 1 Parameters of support hydraulic system

\begin{tabular}{llll}
\hline Quantity & Symbol & Value & Units \\
\hline Area of rod chamber & $A_{c}$ & 0.411 & $\mathrm{~m}^{2}$ \\
Damping coefficient & $B_{V}$ & 200 & $\mathrm{~N} \cdot \mathrm{s} / \mathrm{m}$ \\
Piston diameter & $d$ & 0.915 & $\mathrm{~m}$ \\
Seat diameter & $D_{\mathrm{s}}$ & 0.02 & $\mathrm{~m}$ \\
Friction force of cylinder & $F_{\mathrm{fc}}$ & 500 & $\mathrm{~N}$ \\
Spring stiffness & $k$ & 15 & $\mathrm{kN} / \mathrm{m}$ \\
Piston length & $I_{\mathrm{c}}$ & 0.26 & $\mathrm{~m}$ \\
Cylinder body mass & $m_{\mathrm{C}}$ & 2000 & $\mathrm{~kg}$ \\
Valve mass & $m_{\mathrm{v}}$ & 0.5 & $\mathrm{~kg}$ \\
Supply pressure & $p_{\mathrm{s}}$ & 23 & $\mathrm{MPa}$ \\
Kinematic viscosity & $v$ & 20 & $\mathrm{~mm} / \mathrm{s}$ \\
Volume of rod chamber & $V$ & 0.123 & $\mathrm{~m}$ \\
Spring pre-compression & $y_{0}$ & 0.015 & $\mathrm{~m}$ \\
Fluid density & $\rho$ & 870 & $\mathrm{~kg} / \mathrm{m}^{3}$ \\
Absolute viscosity & $\mu$ & 0.017 & $\mathrm{~Pa} \cdot \mathrm{s}$ \\
Clearance of & $\delta_{1}$ & 0.01 & $\mathrm{~mm}$ \\
Half cone angle & $\theta$ & 30 & $\left({ }^{\circ}\right)$ \\
Bulk modulus & $\beta_{\mathrm{e}}$ & 1.7 & $\mathrm{GPa}$ \\
\hline & & & \\
\hline
\end{tabular}

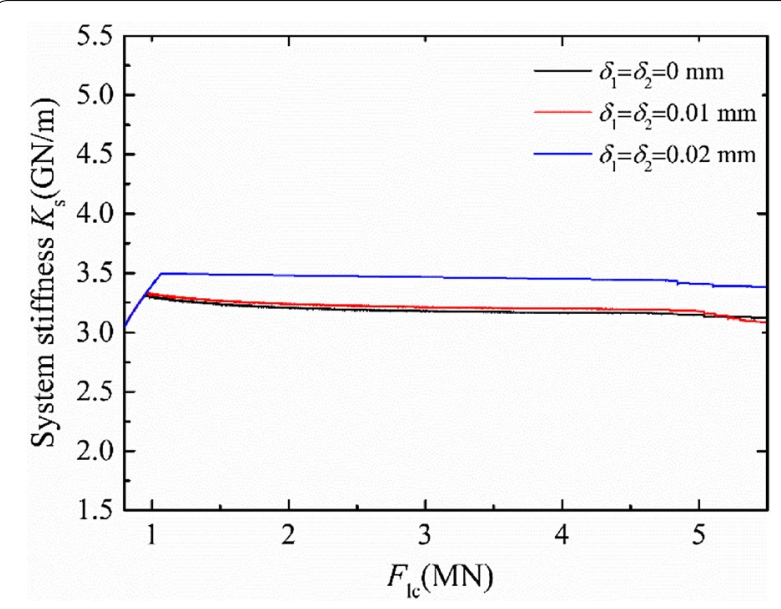

Figure 9 Static stiffness characteristic at $\delta_{1}=\delta_{2}$

The static stiffness increases as the clearances between the piston and the internal wall of the cylinder increase because the fluid working media from the rod-less chamber to the rod one increases the pressure in the rod chamber.

The decrease point of the three stages occurs at about 4.8 MN. At that point, the relief valve connected to the rod chamber of the left cylinder opens, decreasing the static stiffness of the support hydraulic system.

For $\delta_{1} \neq \delta_{2}$, the static stiffness characteristic of the support hydraulic system under different conditions is illustrated in Figure 10.

As illustrated in Figure 10, the clearance of the left cylinder influences the static stiffness of the support

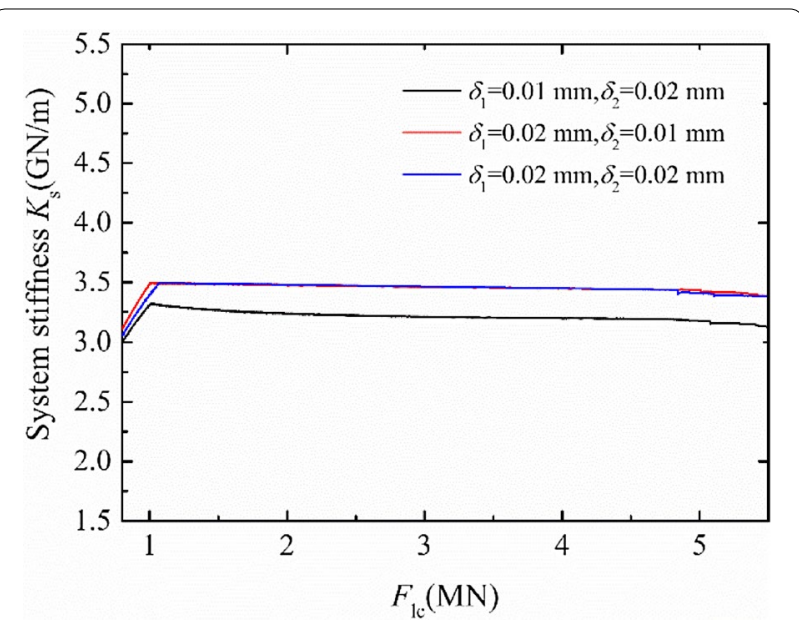

Figure 10 Static stiffness characteristic at $\delta_{1} \neq \delta_{2}$

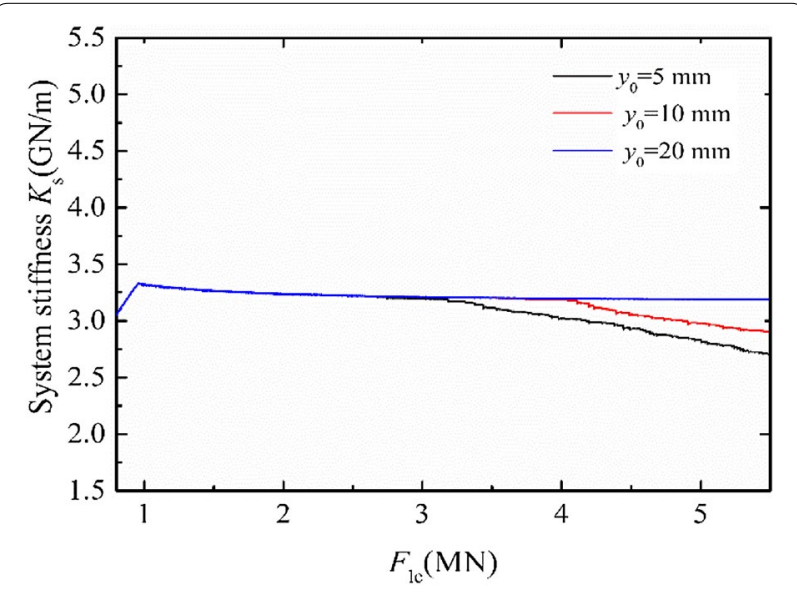

Figure 11 Static stiffness characteristic with different pre-compression lengths at $\delta_{1}=\delta_{2}=0.01 \mathrm{~mm}$

hydraulic system, while the clearance of the right cylinder has little influence on the static stiffness because the working fluid media from the rod-less chamber to the rod one increases the pressure inside the rod chamber of the left cylinder, which provides a counter-force against the external force acting on the cylinder.

\subsubsection{Influence of the Pre-Compression Spring Length-in the Relief Valve on Dynamic Stiffness}

The static stiffness characteristics with different pre-compressions length of the spring are analyzed, and Figure 11 shows the stiffness characteristics of the support hydraulic system at $\delta_{1}=\delta_{2}=0.01 \mathrm{~mm}$.

As shown in Figure 11, the pre-compression length of the spring in the relief valve has no influence on the stiffness value of the increase and stable stages. The range of the stable stage increases as the pre-compression length 


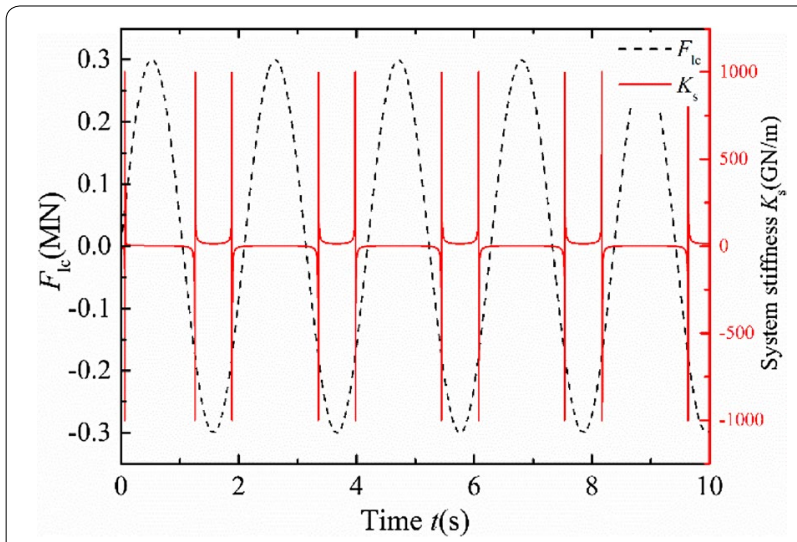

Figure 12 Dynamic stiffness characteristics

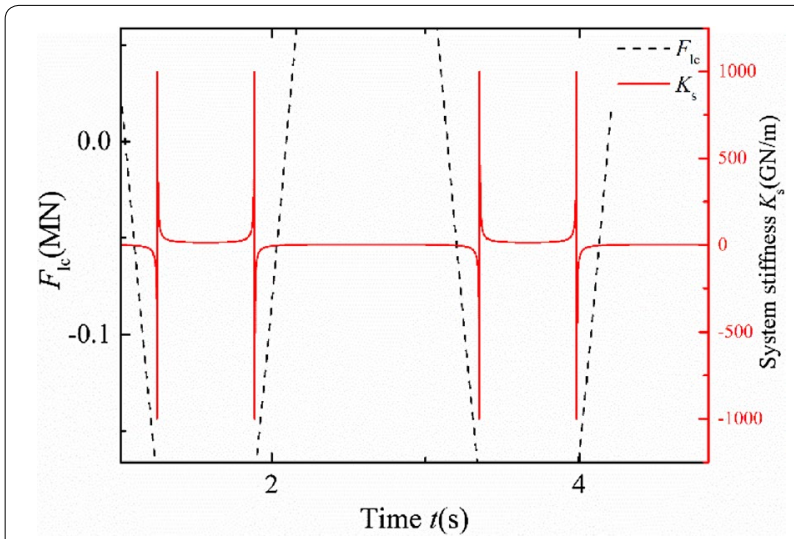

Figure 13 Partial enlarged view of dynamic stiffness characteristics

of the spring increases because this also increases the cracking pressure of the relief valve. The pressure inside the rod chamber of the left cylinder decreases when the connected relief valve opens; therefore, the stiffness of the support hydraulic system also decreases.

It must be noted that the output force of the gripper cylinder decreases when the cracking pressure increases when the supply pressure of the support hydraulic system is kept constant.

\subsection{Dynamic Stiffness Characteristics Analysis}

Figure 12 shows the nonlinear dynamic stiffness characteristics of the support hydraulic system when the external force acting on the cylinder body takes the form of $F_{\mathrm{lc}}=a \sin \omega t, a=0.3 \mathrm{MN}$, and $\omega=3 \mathrm{rad} / \mathrm{s}$, and Figure 13 is a partial enlarged view of Figure 12.

As seen in Figures 12 and 13, the dynamic stiffness switches between the positive and negative extreme values when the direction of the displacement changes, and the dynamic stiffness of the support hydraulic system

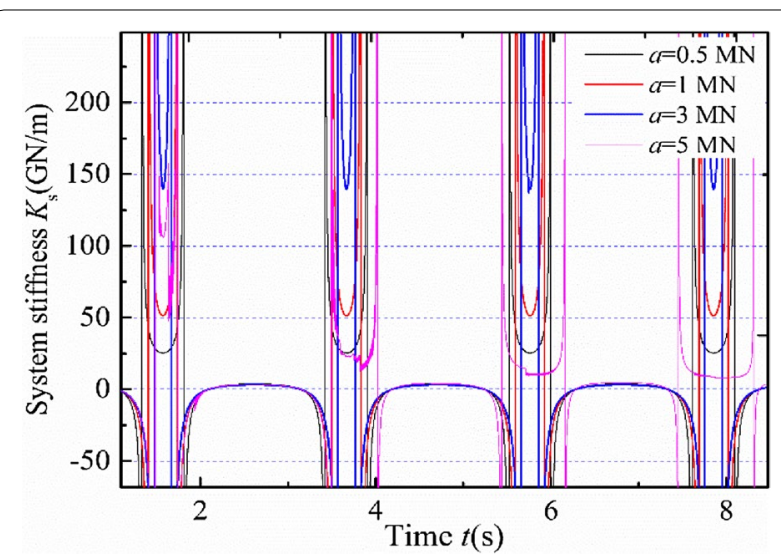

Figure 14 Dynamic stiffness characteristics at different amplitudes

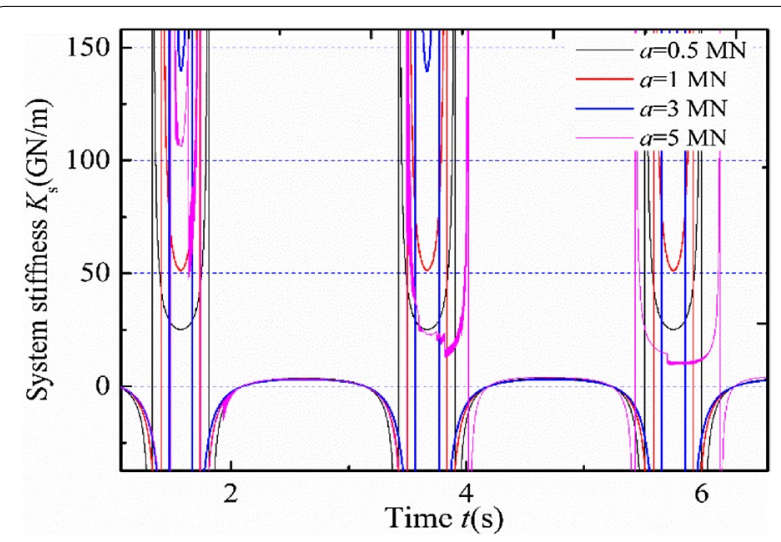

Figure 15 Partial enlarged view of dynamic stiffness characteristics at different amplitudes

takes on a U-shape and reversed U-shape, respectively. The U-shape represents the displacement of the cylinder body in the opposite direction of $x$, and the reversed U-shape denotes the displacement of the cylinder body in the direction of $x$. The bottom value of the U-shape is about $150 \mathrm{GN} / \mathrm{m}$, and the top value of the reversed $\mathrm{U}$-shape is about $3.9 \mathrm{GN} / \mathrm{m}$.

\subsubsection{Influence of the External Force Amplitude Acting on the Cylinder Body}

The external force acting on the cylinder body takes the form of $F_{l c}=a \sin \omega t$ in which $\omega=3 \mathrm{rad} / \mathrm{s}$, and $a$ takes on the values $0.5 \mathrm{MN}, 1 \mathrm{MN}, 3 \mathrm{MN}$, and $5 \mathrm{MN}$. Figure 14 shows the nonlinear dynamic stiffness characteristics of the support hydraulic system at different amplitudes of the force. Figure 15 is the partial enlarged view of Figure 14.

As shown in Figures 14 and 15, the top values of the reversed U-shapes remain constant with external force 


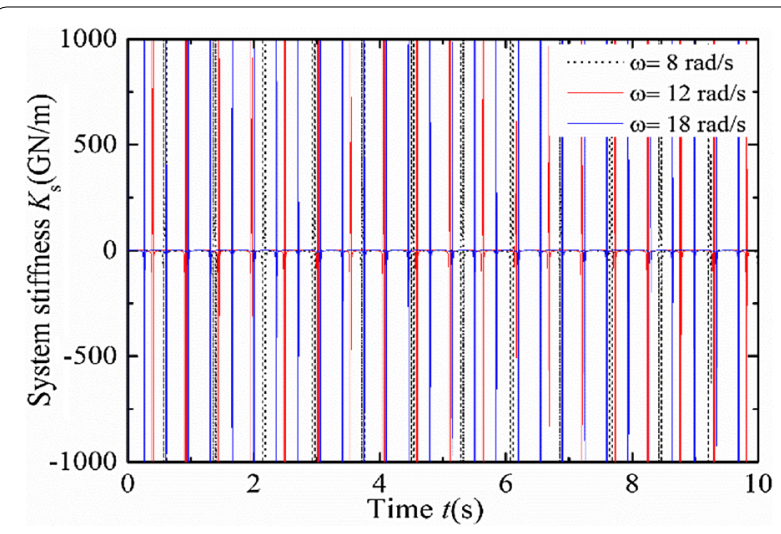

Figure 16 Dynamic stiffness characteristics at different frequencies

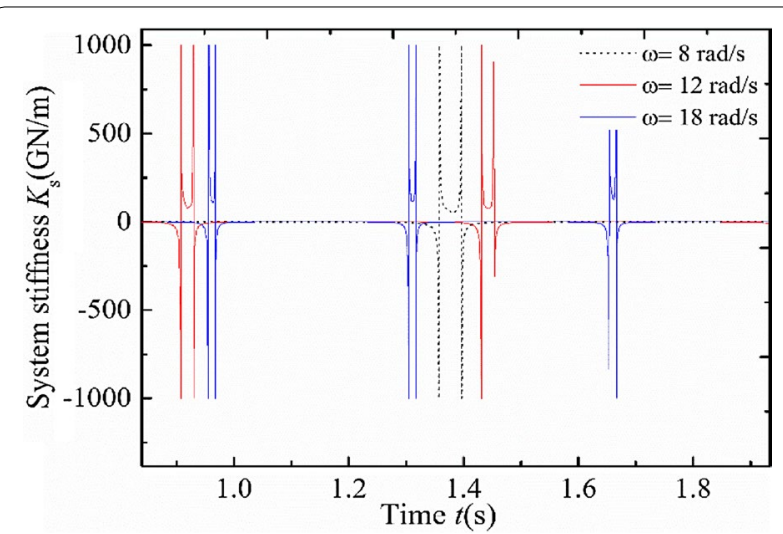

Figure 17 Partial enlarged view of dynamic stiffness characteristics at different frequencies

amplitudes. The bottom value of the U-shape increases and the width decreases as the amplitude of the external force increases until $a=3 \mathrm{MN}$. When the amplitude is $5 \mathrm{MN}$, the bottom value of the U-shape decreases and the width increases because the relief valve opens and the pressure in the rod chamber decreases.

\subsubsection{Influence of the External Force Frequency Acting on the Cylinder Body}

The external force acting on the cylinder body takes the form $F_{l c}=a \sin \omega t$ in which $a=0.3 \mathrm{MN}$, and $\omega$ takes on the values $8 \mathrm{rad} / \mathrm{s}, 12 \mathrm{rad} / \mathrm{s}$, and $18 \mathrm{rad} / \mathrm{s}$. Figure 16 illustrates the nonlinear dynamic stiffness characteristics when the external force acts on the cylinder body with different frequencies. Figure 17 is the partial enlarged view of Figure 16.

As shown in Figures 16 and 17, the bottom value of the U-shape increases as the frequency of the external force acting on the cylinder body increases, and the top value of the reverse U-shapes remain constant with varying frequencies. The top value of the U-shape is about $3.12 \mathrm{GN} / \mathrm{m}$.

\section{Conclusions}

In this study, the nonlinear dynamic model of the support hydraulic system in TBM is established, and the nonlinear static and dynamic stiffness characteristics of the support hydraulic system are analyzed. Based on the analysis results, several conclusions can be obtained.

(a) The static stiffness of the support hydraulic system consists of three stages; the increase stage, stable stage, and decrease stage.

(b) The static stiffness of the support hydraulic system increases as the clearances between the piston and the internal wall of the gripper cylinder increase.

(c) The pre-compression length of the spring in the relief valve has no influence on the static stiffness value of the support hydraulic system; however, the pre-compression length does influence the range of the stable stage of the static stiffness.

(d) The dynamic stiffness of the support hydraulic system consists of a U-shape and reverse U-shape.

(e) The bottom value of the U-shape increases as the amplitude of the external force acting on the cylinder body increases, while the top value of the reverse remains $U$-shape constant.

(f) The bottom value of the U-shape increases as the frequency of the external force acting on the cylinder body increases, while the top value of the reverse U-shape remains constant.

\section{Authors' Contributions}

$J L$ was in charge of the whole trial and established the numerical model of the relief valve; JT established the numerical model of support system and wrote the manuscript; CL assisted with data analyses and WY established the simulation model of the support hydraulic system of TBM with Simulink. All authors read and approved the final manuscript.

\section{Authors' Information}

Jianfeng Tao, born in 1975, is currently an associate professor at Shanghai Jiaotong University, China. He received his PhD degree on mechatronics from Beijing University of Aeronautics and Astronautics, China, in 2003. His research interests include mechatronics engineering, fluid power transmission and control and robotics.

Junbo Lei, born in 1980, is currently a research assistant at Shanghai Jiaotong University, China. He received his PhD degree on mechatronics from Zhejiang University, China, in 2012. His research interests include mechatronics engineering, fluid power transmission and control, and hydraulic component design.

Chengliang Liu, born in 1964, is currently a professor and a PhD candidate supervisor at Shanghai Jiaotong University, China. He received his PhD degree from Southeast University, China, in 1999. His research interests include mechatronics engineering, man-machine system and robotics.

Wei Yuan, born in 1981, is currently an associate professor at Jiangsu University of Science and Technology, China. He received his master degree on control engineering from Jiangsu University of Science and Technology, China, in 
2007. His research interests include nonlinear control systems, robust control and convex optimization.

\section{Competing Interests}

The authors declare that they have no competing interests.

\section{Funding}

Supported by National Key R\&D Program of China (Grant No. 2018YFB1702503), National Program on Key Basic Research Project of China (973 Program, Grant No. 2013CB035403) and Startup Fund for Youngman Research at SJTU (SFYR at SJTU)

\section{Author Details}

${ }^{1}$ School of mechanical engineering, Shanghai Jiaotong University, Shanghai 200240, China. ${ }^{2}$ School of Electronic Information, Jiangsu University of Science and Technology, Zhenjiang 212003, China.

Received: 15 February 2019 Accepted: 4 November 2019 Published online: 05 December 2019

\section{References}

[1] Q S Liu, X Huang, Q M Gong, et al. Application and development of hard rock TBM and its prospect in China. Tunnelling and Underground Space Technology, 2016, 57: 33-46. doi: https://doi.org/10.1016/j. tust.2016.01.034.

[2] J Z Huo, W Z Wang, W Sun, et al. The multi-stage rock fragmentation load prediction model of tunnel boring machine cutter group based on dense core theory. International Journal of Advanced Manufacturing Technology, 2016: 1-13.

[3] W Sun, Jingxiu Ling, J Huo, et al. Study of TBM cutterhead fatigue damage mechanisms based on a segmented comprehensive failure criterion. Engineering Failure Analysis, 2015, 58, Part 1: 64-82. doi: http://dx.doi. org/10.1016/j.engfailanal.2015.08.041.

[4] J Z Huo, HYWu, J Yang, et al. Multi-directional coupling dynamic characteristics analysis of TBM cutterhead system based on tunnelling field test. Journal of Mechanical Science and Technology, 2015, 29 (8): 3043-3058. https://doi.org/10.1007/s12206-015-0701-1.

[5] J Z Huo, N Hou, W Sun, et al. Analyses of dynamic characteristics and structure optimization of tunnel boring machine cutter system with multi-joint surface. Nonlinear Dynamics, 2016: 1-18.

[6] J Z Huo, D Zhu, N Hou, et al. Application of a small-timescale fatigue, crack-growth model to the plane stress/strain transition in predicting the lifetime of a tunnel-boring-machine cutter head. Engineering Failure Analysis, 2016, 71: 11-30.

[7] XY Zou, H Zheng, Y Z Mi. Performance evaluation of hard rock TBMs considering operational and rock conditions. Shock and Vibration, 2018, 2018: 1-17. https://doi.org/10.1155/2018/8798232.

[8] THuang, X L Wang, H T Liu, et al. Force analysis of an open TBM grippingthrusting-regripping mechanism. Mechanism and Machine Theory, 2016, 98: 101-113. doi: https://doi.org/10.1016/j.mechmachtheory.2015.12.003.

[9] Y H Yang, G Chen, X Z Liu, et al. Sensitivity analysis of an open TBM gripping-thrusting-regripping mechanism based on the natural characteristics. Mechanism and Machine Theory, 2019, 139: 1-20. doi: https://doi. org/10.1016/j.mechmachtheory.2019.04.006.

[10] Q J Xie, H D Yu. Coupling relationship between loads on cutterhead of tunnel boring machine and contact stiffness of gripper shoes and rocks. Journal of Shanghai Jiaotong University, 2015, 49(9): 1269-1275.

[11] P Hao, H D Yu, Y Zhao. Normal stiffness of tunnel surface contacting with thrusting boots of TBM with various surface characteristics. Journal of Shanghai Jiaotong University, 2014, 48(6): 827-832.

[12] A Alsahly, J Stascheit, G Meschke. Advanced finite element modeling of excavation and advancement processes in mechanized tunneling.
Advances in Engineering Software, 2016, 100: 198-214. doi: https://doi. org/10.1016/j.advengsoft.2016.07.011.

[13] Y Zhao, H Pan, H Wang, et al. Dynamics research on grouping characteristics of a shield tunneling machine's thrust system. Automation in Construction, 2017, 76: 97-107. doi: https://doi.org/10.1016/j.autco n.2016.12.004.

[14] HY Wu, J Z Huo, Z C Meng, et al. Load characteristics study with a multi-coupling dynamic model for TBM supporting system based on a field strain test. Tunnelling and Underground Space Technology, 2019, 91: 103016. doi: https://doi.org/10.1016/j.tust.2019.103016.

[15] H D Yu, P Hao, Y Zhao, et al. Non-linear behavior of normal contact stiffness of tunnel surface and supporting system of tunnel boring machines. Journal of Mechanical Engineering, 2014, 50(21): 54-59. (in Chinese)

[16] HYYang, H Shi, G F Gong, et al. Electro-hydraulic proportional control of thrust system for shield tunneling machine. Automation in Construction, 2009, 18(7): 950-956. doi: http://dx.doi.org/10.1016/j.autcon.2009.04.005.

[17] R A Snowdon, M D Ryley, J Temporal, et al. The effect of hydraulic stiffness on tunnel boring machine performance. International Journal of Rock Mechanics and Mining Sciences \& Geomechanics Abstracts, 1983, 20(5): $203-$ 214. doi: http://dx.doi.org/10.1016/0148-9062(83)90001-3.

[18] Y J Wu, J F Tao, C L Liu. Modeling on stiffness of gripper in hardrock TBM. 2016 12th IEEE/ASME International Conference on Mechatronic and Embedded Systems and Applications (MESA), 2016: 1-6. https://doi.org/10.1109/ mesa.2016.7587131.

[19] L H Wang, B Wu, R S Du, et al. Nonlinear dynamic characteristics of moving hydraulic cylinder. Journal of Mechanical Engineering, 2007, 43(12): 12-19. (in Chinese)

[20] J B Lei, J F Tao, C L Liu, et al. Internal leakage characteristics of gripper cylinder of TBM. 2016 12th IEEE/ASME International Conference on Mechatronic and Embedded Systems and Applications (MESA), 2016: 1-5. https://doi. org/10.1109/mesa.2016.7587109.

[21] C J Hős, A R Champneys, K Paul, et al. Dynamic behaviour of direct spring loaded pressure relief valves connected to inlet piping: IV review and recommendations. Journal of Loss Prevention in the Process Industries, 2017, 48: 270-288. doi: https://doi.org/10.1016/j.jp.2017.04.005.

[22] Y Gorash, W Dempster, W D Nicholls, et al. Study of mechanical aspects of leak tightness in a pressure relief valve using advanced FE-analysis. Journal of Loss Prevention in the Process Industries, 2016, 43: 61-74. doi: https:// doi.org/10.1016/j.jlp.2016.04.009.

[23] J F Liao, X C Zhu, B Yao. Dynamic modeling of gripper type hard rock tunnel boring machine. Tunnelling and Underground Space Technology, 2018, 71: 166-179. doi: https://doi.org/10.1016/j.tust.2017.08.003.

[24] G Chen, Y H Yang, THuang. Vibration analysis of open TBM grippingthrusting-regripping mechanism. Mechanism and Machine Theory, 2019, 134: 95-116. doi: https://doi.org/10.1016/j.mechmachtheory.2018.12.028.

[25] C J Shao, J F Liao, ZT Liu, et al. Indirect adaptive robust trajectory tracking control of hard rock TBM with load variation of tunneling face. Chinese Journal of Mechanical Engineering, 2019, 32(1): 34. https://doi.org/10.1186/ s10033-019-0347-5.

[26] N Manring. Hydraulic control systems. 1st ed. New Jersey: Wiley, 2005.

[27] F M White. Fluid mechanics. 7th ed. McGraw-Hill, 2009.

[28] [28] J B Lei, J F Tao, C L Liu, et al. Flow model and dynamic characteristics of a direct spring loaded poppet relief valve. Proceedings of the Institution of Mechanical Engineers, Part C: Journal of Mechanical Engineering Science, 2018, 232(9): 1657-1664. https://doi.org/10.1177/0954406217707546.

[29] H E Merritt. Hydraulic control system. New York: John Wiley and Sons, 1967.

[30] C Bazsó, C J Hős. An experimental study on the stability of a direct spring loaded poppet relief valve. Journal of Fluids and Structures, 2013, 42: 456465. doi: http://dx.doi.org/10.1016/j.jfluidstructs.2013.08.008.

[31] K Kasai. On the stability of a poppet valve with an elastic support: 1st Report, Considering the effect of the inlet piping system. Bulletin of JSME, 1968, 11 (48): 1068-1083. https://doi.org/10.1299/jsme1958.11.1068. 